\title{
Presente y futuro de la publicación y difusión del conocimiento
} jurídico

\section{Present and future of the publication and dissemination of legal knowledge}

Bardales-del Aguila, Lionel ${ }^{1}[0000-0002-9110-4475]$

${ }^{1}$ Universidad Nacional de San Martín, Tarapoto, Perú

\section{Editorial}

lbardaleseunsm.edu.pe

El sistema educativo universitario peruano con la Ley Universitaria 30220 (MINEDU, 2015) rediseña su modelo educativo, en el que predomina la investigación científica. La Universidad Nacional de San Martín (UNSM) en el marco de la adecuación a la Ley, ha reformado su estatuto, reglamentado y aprobado normas sobre gestión de la investigación.

Las exigencias del nuevo contexto, han sido asumidas por autoridades, docentes y estudiantes; esto se aprecia con la obtención del licenciamiento institucional, proceso obligatorio monitoreado por la Superintendencia Nacional de Educación Superior Universitaria (SUNEDU) que establece el cumplimiento de las Condiciones Básicas de Calidad (CBC) (Sunedu, 2019) como requisitos mínimos para brindar el servicio educativo de calidad en la formación de profesionales altamente calificados, que respondan a las necesidades y demandas sociales que permita mejorar las condiciones de vida de la población.

Este proceso, no es concluyente; hoy marchamos hacia la acreditación de los programas de estudio (SINEACE, 2018), que exige el cumplimiento de estándares de calidad establecidos en el "Modelo de acreditación para programas de estudios de educación superior universitaria" establecidos por el (Sistema Nacional de Evaluación, 2017) - SINEACE- en los estándares 22, 23 y 24 referidos a la Investigación, Desarrollo Tecnológico e Investigación (I+D+i).

El II Informe Bienal Sobre la Realidad Universitaria en el Perú, 2020 (SUNEDU, 2020) evidencian el avance de la UNSM; apareciendo en los rankings de producción científica, lo cual es corroborado por el estudio de (Valles-Coral et al., 2020) donde la UNSM pasó del puesto 64 en el año 2018 hasta el 44 en el 2020 (Aguillo, 2020), mejorando 20 posiciones; siendo la universidad nacional que mejor desempeño ha mostrado en ese periodo.

Es así que el Vice Rectorado de Investigación en coordinación estrecha con el Fondo Editorial, la Unidad de Investigación, la Decanatura de la Facultad de Derecho y Ciencias Políticas; pese a las condiciones restrictivas impuestas por la pandemia del Covid-19 ha venido trabajando en la creación y gestación de la Revista Científica Ratio Iure en versión electrónica que cumple con las exigencias para lograr su indexación en índices importantes como DOAJ, Redalyc, Latindex Directorio y Scielo.

Ponemos a disposición del público en general el volumen 1, número 1, de la Revista Científica Ratio Iure; donde docentes y estudiantes publicamos los resultados de los trabajos de 
investigación en derecho y justicia social a fin de que sean revisados y reciban la crítica necesaria para su mejora.

Esperando el impacto deseado y augurando buenos resultados para que la Revista Científica Ratio Iure logre la sostenibilidad y periodicidad necesarias y con ello se mejore el posicionamiento de la Universidad Nacional de San Martín.

Universitariamente.

\section{Dr. Lionel Bardales del Águila}

Director de la Unidad de Investigación -FDyCP

Universidad Nacional de San Martín

\section{Referencias bibliográficas}

Aguillo, I. (2020). Peru | Ranking Web of Universities: More than 28000 institutions ranked. http://www.webometrics.info/en/Latin_America/Peru

MINEDU. (2015). Ley Universitaria 30220 (p. 35). MINEDU. http://www.minedu.gob.pe/reforma-universitaria/pdf/ley_universitaria.pdf

SINEACE. (2018). Acreditación. Sistema Nacional de Evaluación. https://www.sineace.gob.pe/acreditacion/

Sistema Nacional de Evaluación, A. y C. de la C. E.-S. (2017). Modelo de acreditación para programas de estudios de educación superior universitaria.

Sunedu. (2019). Condiciones básicas de calidad. 2019. https://www.sunedu.gob.pe/8condiciones-basicas-de-calidad/

SUNEDU. (2020). II Informe Bienal sobre la Realidad Universitaria en el Perú | Gobierno del Perú. https://www.gob.pe/institucion/sunedu/informes-publicaciones/1093280-ii-informebienal-sobre-la-realidad-universitaria-en-el-peru

Valles-Coral, M. A., Riascos-Armas, J. O., \& Hernandez-Torres, E. A. (2020). Management of the digital identity of researchers and its effect on the webometrics ranking of a peruvian amazon university. Revista Cubana de Informacion En Ciencias de La Salud, 31(2). https://doi.org/10.36512/rcics.v31i1.1406

Citar como: Bardales del Aguila, L. (2021). Presente y futuro de la publicación y difusión del conocimiento jurídico. Revista Científica Ratio lure, 1(1), 2-3. https://doi.org/10.51252/rcri.v1i1.153 